\title{
Bereitschaftspotential Preceding Eyelid Blinks
}

Isabella Araujo Mota* and Otávio Gomes Lins

Federal University of Pernambuco - UFPE, Recife, PE, Brazil

\begin{abstract}
A Bereitschaftspotential (BP) preceding voluntary eyelid blinks has been described in a few studies. A BP was not found preceding automatic blinks and blepharospasms. BP preceding blinks presents low amplitudes compared to apendicular musculature. A BP preceding eyelid voluntary blinks was demonstrated in patients with early stage Parkinson Disease, with severely reduced amplitude.
\end{abstract}

Keywords: Bereitschaftspotential; Readiness potential; Eyelid blinking

\section{Introduction}

A BP preceding eyelid blinks has only been described in four studies, two of them correlating voluntary blinks and blepharospasm $[1,2]$, one of them evaluating blinking in normal subjects [3] and another evaluating voluntary blinks in patients with Parkinson Disease (PD) [4]. The evaluation of eyelid blinks is important in evaluating neurological and psychiatric diseases which courses with hypo and hyperkinetic abnormalities during the evolution of the disease.

Eyelid blinking is influenced by conscious, unconscious and reflex factors which turn it into and instrument of physiological exploration of neural systems. Spontaneous eye blinking, despite its correlation with levels of attention and mood, presents an unconscious an automatic nature, which probably explains the absence of a BP preceding this motor act, as it has been described in the studies carried out by Mota et al. [3]. Korosec and Pirtosek [1] and Berardelli et al. [2] did not observe a BP preceding blepharospasms. Such fact reinforces the theory that BP precedes only conscious movements, even existing an unconscious and conscious planning of the voluntary motor act $[5,6]$.

The amplitudes of the BP receding eyelid blinks described by MOTA and LINS 2017 were lower than those reported in most studies, since these later are analyzed, in the majority of them, from appendicular musculature, which has larger cortical representation areas $[7,8]$, but similar to the BP preceding other facial muscles movements $[9,10]$. It was not possible the differentiation between early and late component of BP preceding eyelid blinkis, despite a morphologic alteration of the waveform at the latency of -500 ms in MOTA and LINS, 2017 study Onset latencies of the BP preceding voluntary blinks were similar to those described in the literature, starting at about $1700 \mathrm{~ms}$ before the motor act.

The evaluation of patient with Parkinson Disease in early stages revealed a reduction of more than $80 \%$ in the $\mathrm{BP}$ amplitude before voluntary blinks as compared to normal subjects [4]. This is a physiological finding related to the classical signs of bradykinesia of $\mathrm{PD}$, such as reduction of eyelid blinking and hypomimia, suggesting that the damages to the neural systems related to the voluntary blinking probably begin in pre-clinical phases of the disease.

There were not significant changes on the amplitudes of $\mathrm{BP}$ preceding the eyelid blinks in $\mathrm{PD}$ after the use of levodopa in the study carried out by MOTA, CORIOLOANO and LINS, 2017. This finding may be related to involvement of blinking related neural systems even in preclinical and initial phases of the disease or to the involvement of non-dopaminergic systems related to this motor act.

$\mathrm{BP}$ is an interesting neurophysiologic tool to evaluate mechanisms of cortical planning and execution of motor acts by the supplementary premotor area, supplementary motor area and the lateral pre-motor cortex, respectively. There are few studies involving BP alterations preceding eyelid blinks, turning it into an open field for scientific exploration.

\section{References}

1. Korosec M, Pirtosek Z (2000) Bereitschaftspotential preceding voluntary blink in normal subjects and patients with blepharospasm. Neurol Croat 49: 56 .

2. Berardelli A, Rothwell JC, Day BL, Marsden CD (1985) Pathophysiology of blepharospasm and oromandibular dystonia. Brain 108: 593-608.

3. Mota IA, Lins OG (2017) Bereitschaftspotential preceding spontaneous and voluntary eyelid blinks in normal individuals. Clin Neurophysiol 128: 100-105.

4. Mota IA, Coriolano MG, Lins OG (2017) Bereitschaftspotential eyelid blinks in Parkinson's disease. Arq Neuropsiquiatr 2017: 1-7.

5. Deecke L, Kornhuber HH (1978) An electrical sign of participation of the mesia supplementary motor cortex in human voluntary finger movement. Brain Res 159: $473-476$

6. Shibasaki $\mathrm{H}$, Hallet $M$ (2006) What is the bereitschaftspotential? Clin Neurophysiol 117: 2341-2356.

7. Yoshida K, Kaji R, Hamano T, Kohara N, Kimura J, et al. (1999) Cortica distribution of Bereitschaftspotential and negative slope potential preceding mouth-opening movements in humans. Arch Oral Biol 44: 183-190.

8. Fumuro T, Matsuhashi M, Mitsueda T, Inouchi M, Hitomi T, et al. (2013) Bereitschaftspotential augmentation by neuro-feedback training in Parkinson's disease. Clin Neurophysiol 124: 1398-1405.

9. Galgano J, Froud K (2008) Evidence of the voice-related cortical potential: An electroencephalographic study. Neurolmage 41: 1313-1323.

10. McArdle JJ, Mari Z, Pursley RH, Schulz GM, BraunAR (2009) Electrophysiological evidence of functional integration between the language and motor systems in the brain: A study of the speech Bereitschaftspotential. Clin Neurophysiol 120 275-284.

*Corresponding author: Isabella Araujo Mota Fernandes, Neurologist Medica Doctor; Neurologist at the Federal University of Paraiba; Master in Therapy Innovation from the Federal University of Pernambuco-UFPE, Recife, PE, Brazil, Tel: +55(83)998443333; E-mail: isabella_mota@yahoo.com.br

Received July 20, 2017; Accepted July 28, 2017; Published August 04, 2017

Citation: Mota IA, Lins OG (2017) Bereitschaftspotential Preceding Eyelid Blinks. Int J Neurorehabilitation 4: 279. doi: 10.4172/2376-0281.1000279

Copyright: () 2017 Mota IA, et al. This is an open-access article distributed unde the terms of the Creative Commons Attribution License, which permits unrestricted use, distribution, and reproduction in any medium, provided the original author and source are credited. 